\title{
Tecnologias assistivas em saúde bucal para pessoas com deficiência: produção de um abridor de boca para otimizar 0 atendimento odontológico e a higiene bucal.
}

\author{
Assistive technologies in oral health for people with disabilities: production \\ of a mouth opener to optimize dental care and oral hygiene.
}

FARIA DE MORAES, Ana Luisa

Universidade Federal Fluminense, Discente

analuiisamoraes@hotmail.com

MAIA SILVEIRA, Flávia

Universidade Federal Fluminense, Docente

flaviamaia@globo.com

AMADO DE OLIVEIRA, Giuseppe

Universidade Federal Fluminense, Docente

gamado@id.uff.br

\section{RESUMO}

$\mathrm{Na}$ atenção à saúde bucal de pessoas com deficiência (PCD) é necessária a sua análise integral para identificar suas demandas e planejar soluções adaptadas a cada caso, disponibilizando a tecnologia assistiva (TA). O objetivo deste artigo é apresentar uma intervenção ergonomizadora na clínica odontológica, englobando as fases de diagnose e sistematização ergonômica. O método compreendeu a problematização Sistema-Homem-Tarefa-Máquina do dentista no atendimento a PCD, que foram examinados e analisados o contexto de vida e de saúde, limitações e funcionalidades para identificar as demandas de saúde e TA em saúde bucal. Após análise dos casos, o principal problema identificado foi a dificuldade dos pacientes em manter a boca aberta durante as consultas e higiene. Assim, foi iniciada a projeção de um produto que minimize o problema. O projeto tem grande relevância em relação à inclusão social, à pesquisa e à contribuição para segurança e confiança dos alunos durante o atendimento e de PCD.

Ergonomia, Odontologia, Produto, Tecnologia, Assistiva 


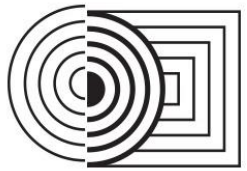

\section{$17^{\circ}$ ERGODESIGN \& USIHC 2019 \\ PUC-Rio, 11 a 13 de dezembro \\ Rio de Janeiro, RJ, Brasil}

$17^{\circ}$ Ergodesign - Congresso Internacional de Ergonomia e Usabilidade de Interfaces Humano Tecnológica: Produto, Informações Ambientes Construídos e Transporte

$17^{\circ}$ USIHC - Congresso Internacional de Ergonomia e Usabilidade de Interfaces Humano Computador

\begin{abstract}
In the oral health care of people with disabilities (PCD) is necessary its full analysis to identify their demands and plan solutions tailored to each case, making available assistive technology (AT). The aim of this paper is to present an ergonomizing intervention in the dental clinic, encompassing the phases of diagnosis and ergonomic systematization. The method comprised the dentist System-Man-Task-Machine problematization in PCD care, which examined and analyzed the context of life and health, limitations and functionalities to identify the demands of health and ED in oral health. After analyzing the cases, the main problem identified was the difficulty of patients to keep their mouths open during consultations and hygiene. Thus, the projection of a product that minimizes the problem was started. The project has great relevance in relation to social inclusion, research and contribution to the safety and confidence of students during attendance and PCD.
\end{abstract}

Ergonomics, Dentistry, Product, Assistive, Technology

\title{
RESUMO EXTENDIDO
}

Aproximadamente $24 \%$ da população brasileira têm algum tipo de deficiência, segundo pesquisas do Censo 2010. O crescimento em relação ao número de pessoas que declarou deficiência ou incapacidade no último Censo Demográfico é enorme. Porém, apesar do crescimento repentino, esses números ainda escondem uma parte da população, pois não se considerou como deficiência mental alguns desvios psíquicos como autismo, esquizofrenia e outras neuroses. Pessoas com deficiências físicas e intelectuais em diferentes níveis são encontradas nas clínicas de saúde bucal todos os dias. A inexistência de utensílios para atendimento específico desses pacientes, é um problema no cotidiano da população. Impõe-se a necessidade de um trabalho em conjunto da equipe de profissionais dentistas e projetistas de produto, caracterizando uma integração interdisciplinar e intersetorial para que com diferentes expertises seja viável desenvolver produtos de tecnologia assistiva de forma bem planejada e com qualidade técnica $A$ tecnologia assistiva (TA) são recursos e dispositivos cujo objetivo é manter ou melhorar a função e a independência de um indivíduo para facilitar a participação social e execução de todas as funções e atividades da vida, contribuindo para o bem-estar geral e qualidade de vida.

A tecnologia assistiva inclui ampla gama de ferramentas, desde um dispositivo simples e de baixa tecnologia, como uma lupa, até um dispositivo complexo de alta tecnologia, como um sistema de comunicação computadorizado. Exemplos de dispositivos e tecnologias assistivas incluem cadeiras de rodas, próteses, bengalas, aparelhos auxiliares de audição, recursos visuais, software e hardware especializados que aumentam a mobilidade, a audição, a visão ou as capacidades de comunicação. Em relação à higiene bucal, além de existirem poucos produtos de TA disponíveis no mercado, não atendem a muitas das demandas de pacientes com problemas motores, comumente observados nos casos de paralisia cerebral e de sequelas de acidente vascular cerebral ou de lesões medulares, características de muitos dos nossos pacientes.

No Brasil a saúde bucal é apontada como a maior necessidade não resolvida das pessoas com deficiência (FOURNIOL,1998), as possíveis explicações são: falta de informação e/ou comprometimento dos responsáveis com relação à saúde bucal; falta de acessibilidade; número restrito e falta de capacitação de profissional, bem como grupos de estudos que discutam métodos facilitadores de prevenção e tratamento odontológico voltados para esses pacientes (VARELLIS, 2005). A maior prevalência de cárie e outras doenças bucais em 


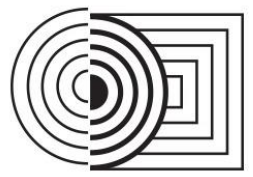

\section{$17^{\circ}$ ERGODESIGN \& USIHC 2019 \\ PUC-Rio, 11 a 13 de dezembro \\ Rio de Janeiro, RJ, Brasil}

$17^{\circ}$ Ergodesign - Congresso Internacional de Ergonomia e Usabilidade

de Interfaces Humano Tecnológica: Produto, Informações Ambientes Construídos e Transporte

$17^{\circ}$ USIHC - Congresso Internacional de Ergonomia e Usabilidade

de Interfaces Humano Computador

pessoas com deficiência normalmente está associada a fatores relacionados à deficiência ou ao uso de medicamentos, como no caso da xerostomia, ou higiene bucal deficiente, por limitação da abertura de boca, movimentos involuntários, incoordenação motora, dentre outros (Anders, Davis, 2010). Embora ainda pouco usado na Odontologia, os recursos tecnológicos de saúde têm demostrado ser opções excelentes, que podem resultar na melhoria da qualidade de vida, na promoção da saúde, na prevenção de deficiências e agravos, influenciando as práticas relacionadas com a reabilitação e a inclusão social dessas pessoas (CALDAS Jr, FRANÇA, 2013).

A manutenção da saúde bucal à pessoa com deficiência depende da construção de vínculos positivos entre a equipe de saúde bucal, o paciente e sua família e a simplificação tecnológica ou adaptação de equipamentos para proporcionar um atendimento mais adequado às necessidades desses pacientes (BERSCH, 2017; BRASIL, 2009a). Para isso, a equipe profissional precisa ouvir a família, entender o contexto de vida do paciente e suas condições de saúde, dificuldades e funcionalidades.

Tecnologia assistiva não é um tema afeto à ciência e tecnologia, à saúde, à indústria, à educação, etc. Na verdade, é um conceito muito mais amplo, um elemento chave para a promoção dos Direitos Humanos, pelo qual as pessoas com deficiência têm a oportunidade de alcançarem sua autonomia e independência em todos os aspectos de suas vidas (BRASIL, 2009). Entretanto, embora ainda não haja números sobre o uso de TA no Brasil, segundo a OMS (WHO, 2015), em muitos países de baixa e média renda, apenas 5 a 15\% das pessoas que necessitam de dispositivos e tecnologias assistivas têm acesso a elas. Portanto, é uma tecnologia extremamente útil, mas ainda muito subutilizada.

O projeto de foi realizado em uma clínica que realiza atendimento odontológico para pessoas com deficiência. Inicialmente foram observados fatores como: movimentação dos pacientes com deficiência durante o atendimento e higiene bucal, capacidade do profissional dentista de realizar seu trabalho com eficiência e problemas ergonômicos envolvidos.

O projeto utiliza as técnicas de análise sistêmica, diagnose, validação e detalhamento da tarefa. Após a análise dos problemas ergonômicos relacionados ao processo, foi gerada uma tabela para definir o problema de maior gravidade a ser trabalhado. Logo após, foi realizada a análise de produtos existentes no mercado com proposta similar à proposta. Foram destacados pontos positivos, negativos e interessantes sobre eles para a realização do modelo final. Para desenvolvimento desse produto, utilizou-se o método da intervenção ergonomizadora, baseado no livro de Anamaria de Moraes, Ergonomia: Conceitos e Aplicações.

Para organizar esses dados, foi construído um relatório de registros para a criação do produto. O principal problema observado foi a dificuldade em manter a boca do paciente aberta durante $o$ atendimento e muitos apresentam estrutura física adulta e sua força inviabiliza que 0 mesmo seja atendido contra sua vontade ou capacidade. Há pacientes solícitos que colaboram com o processo, mas, em algumas ocasiões, sua vontade não basta. Depois de escrita uma modelagem verbal, os requisitos para o projeto foram estabelecidos. A TA produzida precisará garantir estabilidade para o profissional, ser confortável para o usuário, abrir a boca do paciente sem que seja necessária a parada do processo durante a realização da consulta, ser maleável e pouco rígido e obter design anatômico à arcada humana padrão. Os recursos já existentes, não atendem às necessidades em questão e podem machucar. São "dedeiras" e abridores de boca infantil e adulto. A queixa dos profissionais é a remoção do produto toda vez que o paciente move a boca. Os alunos, preferem utilizar as "dedeiras" que garantem que seus dedos não serão mordidos pelos pacientes, contudo, é necessário a utilização de outra mão para auxílio. Seguindo a metodologia do projeto, foi executada uma geração de alternativas (formatos e tamanhos) para o produto mediante a simulação do movimento de abertura da boca 
com manequim odontológico e, dentre elas, uma geração escolhida a partir de uma matriz decisória. Foi realizada uma pesquisa de materiais e processos de fabricação com potencial de uso. O processo de criação segue com a construção de um modelo físico de teste em forma de protótipo para identificar possíveis falhas na usabilidade do produto, elaboração de desenho técnico para a fabricação final e, após o produto pronto, validação e teste de funcionalidade para comprovar sua eficácia.

O intuito do projeto é, além de todas as propostas de utilidade de um produto, promover a ideia de inclusão social contemplando a temática da tecnologia assistiva. Dentro desses objetivos, o projeto busca promover a interação do produto somado à experiência de usabilidade do usuário.

Seguindo a análise de todo o processo, procura-se promover a conscientização de um serviço de saúde bucal prestado à população de forma profissional, segura, com confiabilidade e logística. É desejável que haja a reflexão do tema no meio de projeção de produtos, bem como espera-se um foco maior no mercado específico destinado a pessoas com deficiência, visto que tal tópico não é muito abordado no contexto atual de produção.

\section{REFERÊNCIAS BIBLIOGRÁFICAS}

$\mathrm{BERSCH}, \mathrm{R}$. Introdução à Tecnologia Assistiva. Assistiva. Tecnologia e Educação. Porto Alegre. 2017. Disponível em: http://www.assistiva.com.br/Introducao_Tecnologia_Assistiva.pdf. Acesso em 19 jan. 2019.

BRASIL. Ministério da Saúde. Secretaria de Atenção à Saúde. Departamento de Ações Programáticas Estratégicas. atenção à saúde da pessoa com deficiência no Sistema Único de Saúde - SuS. 1. ed. Brasília: Ministério da Saúde, 2010. Disponível em: Acesso em 19 jan. 2019.

Ministério da Saúde. (2009a). Atenção à Saúde da Pessoa com Deficiência no Sistema Único de Saúde-SUS. Brasília: Ministério da Saúde. Disponível em: http://portal.saude.gov.br/portal/arquivos/pdf/atensaudecomdefic.pdf. Acesso em: 20 jan 2019. (2009b).

CORDE. Subsecretaria Nacional de Promoção dos Direitos da Pessoa com Deficiência. Comitê de Ajudas Técnicas. Tecnologia Assistiva. Brasília, 2009. 138 p.

MORAES, A. ; MONT'ALVÃO, C. . Ergonomia: conceitos e aplicações. 4a․ ed. Rio de Janeiro: 2AB Editora, 2010. BAXTER, M. Projeto de Produto: Guia Prático para o design de novos produtos. São Paulo, Ed. Edgard Blücher,1998.

BENEDETTO, I.L.C. Contribuições Metodológicas para o desenvolvimento de Produtos em Tecnologia Assistiva. Dissertação. PGDesign, UFRGS, Porto Alegre, RS, 2011.

\section{AGRADECIMENTOS}

Ao PROEX, Pró-Reitoria de Extensão pela oportunidade e disponibilidade de bolsa para auxílio na materialização do projeto.

Aos professores e alunos da Clínica do Instituto de Saúde, que, gentilmente compartilharam o espaço e contribuíram, em relatos e demonstrações, para a conclusão do projeto.

Ao professor João Marcos Bittencourt, que orientou parte da problematização do projeto. 
Aos pacientes da Clínica do Instituto de Saúde que concordam e incentivam os projetos acadêmicos junto a seus responsáveis, que permitem a utilização de suas imagens para fins de pesquisa.

Ao voluntário Gabriel de Carvalho Lamblet, que se propôs a testar e ajudar na pesquisa do projeto assim como sua mãe, responsável por sua higiene bucal. 